\title{
Unified Fingerprinting/Ranging Localization for e-Healthcare Systems
}

\author{
Javier Prieto, Juan F. De Paz, Gabriel Villarrubia, Javier Bajo \\ and Juan M. Corchado
}

\begin{abstract}
Indoor localization constitutes one of the main pillars for the provision of context-aware services in e-Healthcare systems. Fingerprinting and ranging have traditionally been placed facing each other to meet the localization requirements. However, accurate fingerprinting may worth the exhaustive calibration effort in some critical areas while easy-to-deploy ranging can provide adequate accuracy for certain non-critical spaces. In this paper, we propose a framework and algorithm for seamless integration of both systems from the Bayesian perspective. We assessed the proposed framework with conventional WiFi devices in comparison to conventional implementations. The presented techniques exhibit a remarkable accuracy improvement while they avoid computationally exhaustive algorithms that impede real-time operation.
\end{abstract}

Keywords Bayesian data fusion $\cdot$ Fingerprinting $\cdot$ Ranging $\cdot$ RSS

\section{Introduction}

The development of a better condition of life for older adults has become a major task over the increasingly aged occidental population [10]. Information and communication technologies are acquiring a major role in this task since they can foster greater quality of life, autonomy and participation in social life of elderly people [4]. Such technologies have resulted in the irruption of a vast range of elderly care [4], home care [5] and e-Healthcare systems [2]. These systems are highly correlated with context, and hence, the knowledge of the location of elderly people can remarkably benefit the provision of these context-aware services [12]. Current posi-

J. Prieto (四) · J.F. De Paz · G. Villarrubia · J.M. Corchado

BISITE Research Group, University of Salamanca, Edificio I+D+i C/ Espejo,

37007 Salamanca, Spain

e-mail: javierp@usal.es

J. Bajo

Departamento de Inteligencia Artificial, Facultad de Informática,

Universidad Politécnica de Madrid, Madrid, Spain 
tioning techniques that rely on global navigation satellite systems (GNSS) provide suitable performances in open areas [6]. However, there is no alternative technique with analogous performance and affordable complexity in harsh environments [11]. The proposed alternatives can be coarsely classified into fingerprinting and ranging localization techniques [12].

Fingerprinting techniques determine the position of a mobile target from locationdependent information provided by offline and online measurements [12]. In the offline phase, different features from the transmitted signals in the wireless network are stored at several locations to form a database of location fingerprints. Then, the position is estimated by comparison of the database with the values received by the target in the online phase (i.e., with its fingerprint). Fingerprinting techniques involve two major drawbacks: they require an arduous calibration offline phase and are very sensitive to fast environmental changes [12].

Ranging techniques determine the position of a mobile target from range-related information provided by received signal-strength (RSS) or time-of-arrival (TOA) measurements [11]. In a first stage, the distance to a set of anchors with known positions is estimated from the signals transmitted to the target. Then, the position is estimated by means of a process known as trilateration (i.e., intersection of circles). Ranging techniques suffer from two dominant limitations: their accuracy is far from fingerprinting methods and falls down under the multipath and non-line-ofsight (NLOS) conditions of harsh environments [6].

Strengths and weaknesses of fingerprinting and ranging localization have inevitably focused the challenge in developing hybrid systems without substantially increasing complexity and cost. Such solutions will enable fine localization via fingerprinting methods in places where the accuracy is critical or the database can be frequently updated, and coarse localization via ranging methods in areas where there is no database or it has become obsolete. In [8] fingerprint- and TOA-based methods are coupled to localize UWB devices from a maximum-likelihood (ML) perspective; in [9] fingerprint- and RSS-based techniques are fused by using RFID tags/readers and the computationally expensive particle filter; whereas in [3] fingerprint-based localization and channel-estimation tracking are combined to localize UWB devices via extended Kalman filter (EKF).

In this paper, we propose a framework for unified fingerprinting/ranging based on Bayesian data fusion. Such framework integrate position-related measurements from the first and range-related measurements from the second. Moreover, it considers the dynamic nature of the target's position and accommodates any other position-related information. We further derive algorithms to implement such framework based on the unscented Kalman filter (UKF) that allows for efficient computation over a Smartphone, facilitating its integration under previously proposed e-Healthcare solutions [12].

The rest of the paper is organized as follows: Sect. 2 exposes the system specification for its integration within an e-Healthcare platform; Sect. 3 presents the framework for unified data fusion of fingerprinting/ranging measurements; Sect. 4 assesses the proposed scheme by an experimental case study; and finally, Sect. 5 summarizes the conclusions drawn from the research. 


\section{System Specification}

This section provides a general overview of the requirements of the localization framework implemented within a previously deployed e-Healthcare system [12].

The localization algorithm will be integrated within a monitoring and tracking platform for people with medical problems [12]. The mentioned platform is based on a virtual organization of agents that monitors user's information. The virtual organization was created with the PANGEA platform that facilitates the development of agents in light devices and the integration of different hardware [14]. Within this platform, the localization role is played in the home care organization. However, the localization algorithm can be executed in the elderly user's device (i.e., in a Smartphone) or in a centralized server (i.e., in the cloud). In the former case, the complexity limitations are imposed by the Smartphone's memory and CPU. In the latter case, the complexity constraints are determined by the number of simultaneous users and the response time. Consequently, we will avoid highly complexity demanding fusion algorithms such as particle filters or Gaussian mixture filters [9, 11].

The monitoring and tracking platform utilizes WiFi as the underlying technology to provide indoor localization. WiFi technology is more accessible and less expensive than other alternative technologies such as RFID or UWB, and has a longer range and larger bandwidth than ZigBee or Bluetooth. From signals transmitted in the WiFi network, we can easily extract the RSS metric, while time- or angle-related measurements imply additional complexities and costs [11]. Hence, we will employ the RSS metric for fingerprinting and ranging.

Figure 1 depicts the flowchart for the whole localization process. In the offline phase, we store the fingerprints for the most critical areas where we desire a more accurate localization. In the online phase, we estimate the position by means of Bayesian data fusion where fingerprinting/ranging models are chosen if the prediction is inside/outside the stored area, respectively. Note that this scheme accommodates other position-related information given by diverse devices such as the Smartphone's GPS receiver or a foot-mounted inertial measurement unit (IMU).

\section{Bayesian Data Fusion}

In this section, we formulate the problem of estimating the position of a mobile agent in a two-dimensional scenario by fusing information from different positionrelated measurements. In order to do that, we collect measurements, $\left\{\mathbf{y}_{k}\right\}_{k \in \mathbb{N}}$, at discrete time instants, $t_{k \in \mathbb{N}}$. From these measurements, we estimate the state vector, $\left\{\mathbf{x}_{k}\right\}_{k \in \mathbb{N}}$. In addition to the information conveyed by the measurements, the fact that the sequence of positions is highly correlated in time can also be used as another source of information. Next, we determine the entries to state and measurement vectors and define the models for the fusion of time-evolution and measuring information. 
The state vector contains the position and its first derivatives so that $\left\{\mathbf{x}_{k}\right\}_{k \in \mathbb{N}}$ is a Markov chain (i.e., the current state only depends on the previous one) $[1,11]$. In this paper, $\mathbf{x}_{k}=\left[\mathbf{p}_{k}, \mathbf{v}_{k}, \mathbf{a}_{k}\right] \in \mathbb{R}^{6}$, where $\mathbf{p}_{k} \in \mathbb{R}^{2}$ is the target's position, $\mathbf{v}_{k} \in \mathbb{R}^{2}$ its velocity, and $\mathbf{a}_{k} \in \mathbb{R}^{2}$ its acceleration. ${ }^{1}$

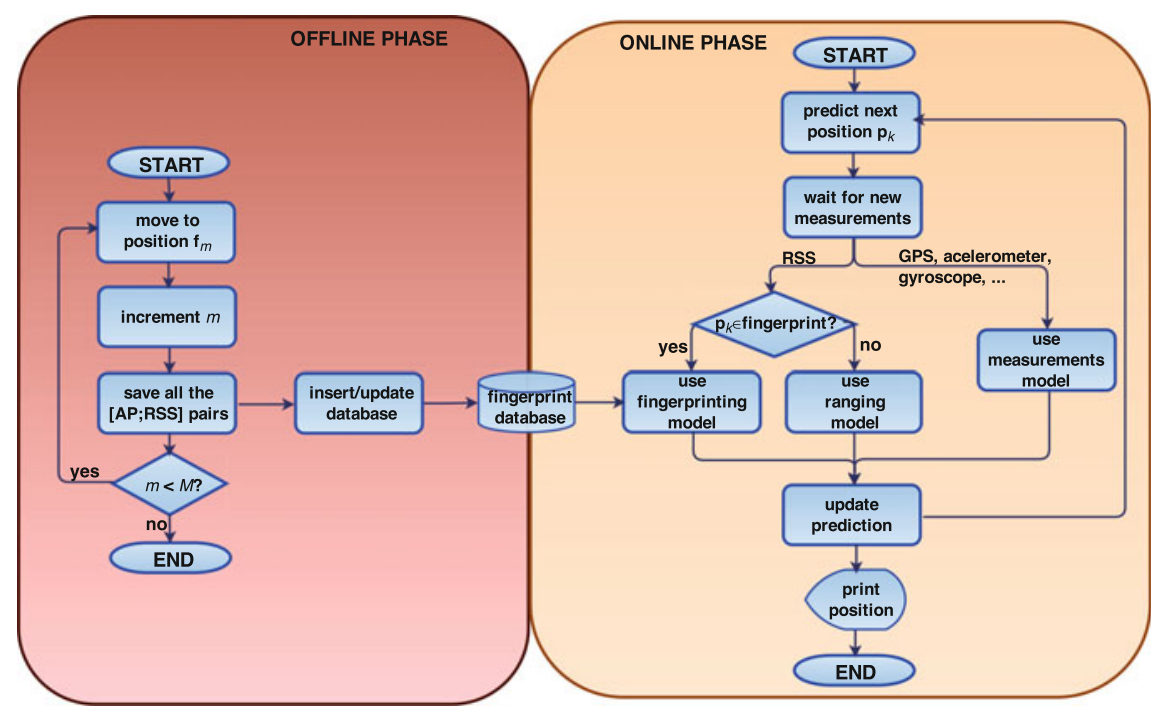

Fig. 1 The proposed localization scheme facilitates a smooth change between fingerprinting and ranging algorithms and the integration of GPS or inertial measurements

The measurement vector conveys any position-related information received at time instant $t_{k}$ (i.e., its dimension may be different to the previous one). In this paper, $\mathbf{y}_{k}=\mathbf{y}_{k}^{f} \in \mathbb{R}^{L_{k}}$, or $\mathbf{y}_{k}=\mathbf{y}_{k}^{s} \in \mathbb{R}^{L_{k}}$, depending on whether we receive the RSS measurements within a critical area (i.e., fingerprinting) or not (i.e., ranging), respectively, where $L_{k} \in \mathbb{R}$ is the number of anchors visible at that particular moment. ${ }^{2}$

With the defined measurements and state vectors, it can be assumed that given the current state vector, $\mathbf{x}_{k}$, the measurement vector, $\mathbf{y}_{k}$, is independent of all previous and future states and measurements [1]. Therefore, we can build a hidden Markov model that leads to two kinds of dependence between the random variables: the relationship between the state vector in time $t_{k}$ and the state vector in time $t_{k-1}$, i.e., $p\left(\mathbf{x}_{k} \mid \mathbf{x}_{k-1}\right)$, called dynamic model; and the relationship between the measurements and the state vector in each time, i.e., $p\left(\mathbf{y}_{k} \mid \mathbf{x}_{k}\right)$, called measurements model $[1,11]$. We define both models in the next subsections.

\footnotetext{
${ }^{1}$ In dead-reckoning systems, it is common to employ a foot-mounted IMU. In this case, the state vector has to be augmented to include the IMU's orientation, its derivatives and measurement biases [13].

${ }^{2}$ In dead-reckoning systems, it is common to gather specific force and angular velocity measurements. In this case, the measurement vector has to be augmented to include their respective values [13].
} 


\subsection{Dynamic Model}

In the following, we define the model that conveys the information provided by the evolution in time of the state vector.

Given the position, velocity and acceleration at time $t_{k-1}, \mathbf{p}_{k-1}, \mathbf{v}_{k-1}$ and $\mathbf{a}_{k-1}$, we can approximate their values in time $t_{k}, \mathbf{p}_{k}, \mathbf{v}_{k}$ and $\mathbf{a}_{k}$, by means of their Taylor series expansion as [1],

$$
\left[\begin{array}{c}
\mathbf{p}_{k} \\
\mathbf{v}_{k} \\
\mathbf{a}_{k}
\end{array}\right]=\left(\begin{array}{ccc}
\mathbf{I}_{2} & \Delta_{k} \mathbf{I}_{2} & \frac{\Delta_{k}^{2}}{2} \mathbf{I}_{2} \\
\mathbf{0} & \mathbf{I}_{2} & \Delta_{k} \mathbf{I}_{2} \\
\mathbf{0} & \mathbf{0} & \mathbf{I}_{2}
\end{array}\right)\left[\begin{array}{c}
\mathbf{p}_{k-1} \\
\mathbf{v}_{k-1} \\
\mathbf{a}_{k-1}
\end{array}\right]+\mathbf{n}_{k}^{d}=\mathbf{F}_{k}\left[\begin{array}{c}
\mathbf{p}_{k-1} \\
\mathbf{v}_{k-1} \\
\mathbf{a}_{k-1}
\end{array}\right]+\mathbf{n}_{k}^{d}
$$

where $\mathbf{I}_{n} \in \mathbb{R}^{n \times n}$ denotes the $n \times n$ identity matrix, $\Delta_{k}=\left(t_{k}-t_{k-1}\right) \in \mathbb{R}$ is the sampling interval, $\mathbf{F}_{k} \in \mathbb{R}^{6 \times 6}$ is the transition matrix, and $\mathbf{n}_{k}^{d} \in \mathbb{R}^{6}$ is the error term where the most common is to model it as white Gaussian noise (i.e., a discrete Wiener process). Therefore, the dynamic model is given by,

$$
p\left(\mathbf{x}_{k} \mid \mathbf{x}_{k-1}\right)=\varphi\left(\mathbf{x}_{k} ; \mathbf{F}_{k} \mathbf{x}_{k-1}, \mathbf{\Sigma}_{k}^{d}\right)
$$

where $\varphi(\mathbf{x} ; \boldsymbol{\mu}, \mathbf{\Sigma})$ denotes the probability density function of a random vector $\mathbf{x} \sim$ $\mathcal{N}(\boldsymbol{\mu}, \boldsymbol{\Sigma})$ and $\boldsymbol{\Sigma}_{k}^{d} \in \mathbb{R}^{6 \times 6}$ is the covariance matrix corresponding to $\mathbf{n}_{k}^{d}$.

\subsection{Measurements Model}

In the following, we describe the models for the relationship between position- or range-related measurements and the state vector.

RSS-Fingerprinting Measurements. We assume that the RSS corresponding to the region of the map associated to fingerprint $\mathbf{f}_{m} \in \mathbb{R}^{2}$ follows a Gaussian distribution. Therefore, the likelihood function of such fingerprint is given by,

$$
p\left(\mathbf{y}_{k}^{f} \mid \mathbf{f}_{m}\right)=\prod_{l=1}^{L_{k}} \varphi\left(y_{k}^{(l)} ; \overline{\mathbf{y}}_{m}^{(l)}, \sigma_{m}^{(l)} / \sqrt{S_{m}^{(l)}}\right)
$$

where $y_{k}^{(l)}$ is an RSS measurement received form the $l$ th anchor in $t_{k}, \overline{\mathbf{y}}_{m}^{(l)}$ is the sample mean of the $S_{m}^{(l)}$ RSS measurements from such anchor stored in the $m$ th fingerprint, and $\sigma_{m}^{(l)}$ is their sample standard deviation. Therefore, we can approximate the measurements model by a mixture of the individual likelihoods at every point of the set $\left\{\mathbf{f}_{m}\right\}_{m=1}^{M}$ as, 


$$
\begin{aligned}
p\left(\mathbf{y}_{k}^{f} \mid \mathbf{p}_{k}\right) & \approx \sum_{m=1}^{M} p\left(\mathbf{y}_{k}^{f} \mid \mathbf{f}_{m}\right) \varphi\left(\mathbf{p}_{k} ; \mathbf{f}_{m}, h^{2} \mathbf{I}_{2}\right) \\
& \approx \sum_{m=1}^{M} \omega_{k}^{(m)} \varphi\left(\mathbf{p}_{k} ; \mathbf{f}_{m}, h^{2} \mathbf{I}_{2}\right)
\end{aligned}
$$

where we have selected a Gaussian kernel with bandwidth $h$ to model each region of the map and approximate the likelihood by a continuous function.

RSS-Ranging Measurements. The RSS values are influenced, among other factors, by the distance between target and anchors. This attenuation is proportional to the inverse of the distance raised to a path-loss exponent [11]. In logarithmic units, we have that for the $l$ th anchor with position $\mathbf{p}^{(l)} \in \mathbb{R}^{2}$,

$$
y_{k}^{s}=\alpha-10 \beta \log _{10}\left\|\mathbf{p}^{(l)}-\mathbf{p}_{k}\right\|+n_{k}^{s}
$$

where $\alpha \in \mathbb{R}$ is a constant that depends on several factors such as fast and slow fading, gains in transmitter and receiver antennas and the transmitted power, and $\beta \in \mathbb{R}$ is the path-loss exponent that can be dynamically obtained or trained in each scenario. Finally, $n_{k}^{s}$ is a Gaussian noise term caused by shadowing [11]. Therefore, the corresponding likelihood function is given by,

$$
p\left(y_{k}^{s} \mid \mathbf{p}_{k}\right)=\varphi\left(y_{k}^{s} ; \alpha-10 \beta \log _{10}\left\|\mathbf{p}^{(l)}-\mathbf{p}_{k}\right\|, \sigma_{k}^{p}\right)
$$

where $\sigma_{k}^{s} \in \mathbb{R}$ is the standard deviation corresponding to $n_{k}^{s}$.

\section{Performance Evaluation}

The goal of this section is to quantify the performance of the localization framework described in Sect. 3 that may help to a better monitoring and tracking of elderly people. The system is evaluated in the experimental case study of a pedestrian walking with a Smartphone that collects RSS measurements from the WiFi network. In the following, we describe the set-up for the experiments and present the performance results.

\subsection{Experimental Set-Up}

To obtain the localization results we utilized dynamic and measurements models described in Sect. 3. ${ }^{3}$ The complexity constraints mentioned in Sect. 2 led to the election of a Kalman-like solution [7]. The lack of linearity in the models implied the use of a suboptimal solution, where the most common is the EKF. For rang-

\footnotetext{
${ }^{3}$ We also added zero-mean Gaussian priors for velocity and acceleration.
} 
ing localization, we selected the UKF since it better captures higher order moments caused by non-linearities and avoids computation of Jacobian and Hessian matrices [7]. For fingerprinting localization, we predicted via Kalman filter (KF) and updated by multiplying the prediction with the Guassian mixture likelihood given by (4) and approximating the result by a single Gaussian.

The mobile target was a pedestrian with a Smartphone that covered the path shown in Fig. 2b with several people walking around. The total length of the path was approximately $110 \mathrm{~m}$, implying a total time of $2.5 \mathrm{~min}$. For fingerprinting localization, the database was created by storing at least 10 RSS values from all the detectable access points (up to 25) in the fingerprints marked in Fig. 2a. For the ranging localization, we employed 16 RSS measurements per point from the 4 access points plotted in Fig. 2a. All the RSS measurements were considerably affected by NLOS and multipath propagation conditions.

We compare our results against a conventional implementation based on Bayesian networks for fingerprinting [2] and ML for ranging [11].

\subsection{Results and Discussion}

Figure $2 \mathrm{~b}$ shows the localization results in the mentioned path. For the proposed approach, the root-mean-square-error (RMSE) was $0.79 \mathrm{~m}$ in fingerprinting, $4.04 \mathrm{~m}$ in ranging, and $2.55 \mathrm{~m}$ in total. For the conventional approach, the RMSE was $1.48 \mathrm{~m}$ in fingerprinting, $5.88 \mathrm{~m}$ in ranging, and $3.79 \mathrm{~m}$ in total.

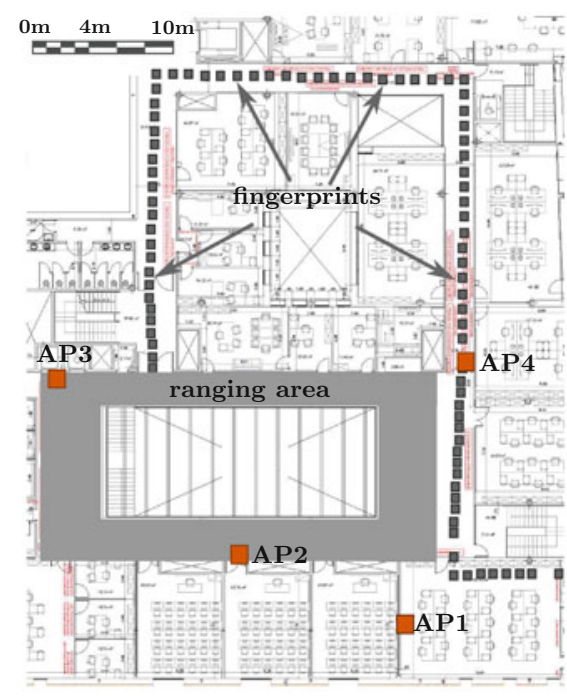

(a) Experimental set-up

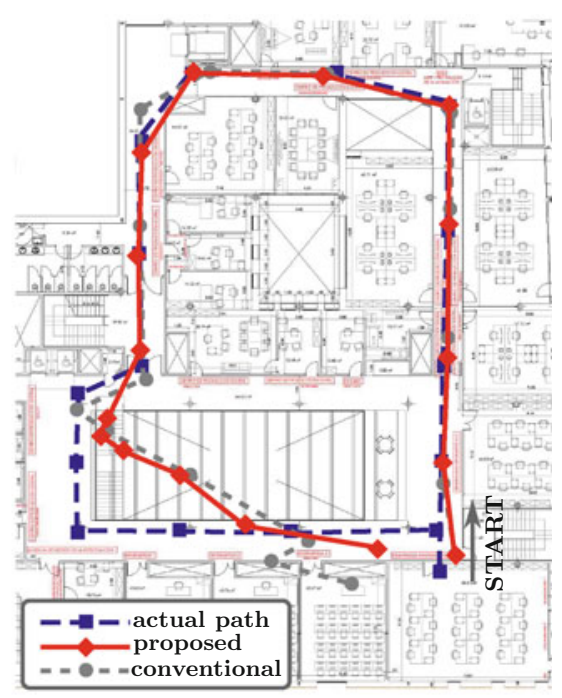

(b) Localization results

Fig. 2 The proposed unified framework provides accurate localization via fingerprinting for critical areas and ready-to-use localization via ranging for non-critical spaces 
From Fig. 2 we can point out that: (1) the proposed framework facilitates the shift from accurate fingerprinting to coarse ranging; (2) the Bayesian approach noticeably improves $\mathrm{ML}$ in ranging localization; and (3) fingerprinting outperforms ranging in WiFi networks while requiring greater calibration effort.

\section{Conclusion}

This paper has presented a principled framework and efficient algorithm for unifying fingerprinting and ranging localization in e-Healthcare systems. We have defined the framework from the Bayesian perspective that allows for the inclusion of positionrelated information coming from heterogeneous sources. We have implemented the algorithm via UKF that holds promise for effective fingerprinting/ranging fusion without a substantial increment in complexity. Under NLOS and multipath conditions, the presented techniques obtained an error in position estimation of $2.5 \mathrm{~m}$ along a 110-meter-long path, remarkably outperforming conventional fingerprinting/ranging implementations.

Acknowledgments This work has been supported by the Spanish Government through the project iHAS (grant TIN2012-36586-C01/C02/C03) and FEDER funds.

\section{References}

1. Y. Bar-Shalom, X.R. Li, T. Kirubarajan, Estimation with Applications to Tracking and Navigation: Theory Algorithms and Software (Wiley, New York, 2001)

2. N. Van den Berg, M. Schumann, K. Kraft, W. Hoffmann, Telemedicine and telecare for older patients-a systematic review. Maturitas 73(2), 94-114 (2012)

3. S. Bybordi, L. Reggiani, Hybrid fingerprinting-EKF based tracking schemes for indoor passive localization. Int. J. Distrib. Sens. Netw. 2014, 1-11 (2014)

4. J.M. Corchado, J. Bajo, A. Abraham, GerAmi: improving healthcare delivery in geriatric residences. IEEE Intell. Syst. 23(2), 19-25 (2008)

5. J.A. Fraile, Y. de Paz, J. Bajo, J.F. de Paz, B.P. Lancho, Context-aware multiagent system: planning home care tasks. Knowl. Inf. Syst. 40(1), 171-203 (2014)

6. F. Gustafsson, F. Gunnarsson, Mobile positioning using wireless networks. IEEE Signal Process. Mag. 22(4), 41-53 (2005)

7. S. Julier, J. Uhlmann, H.F. Durrant-Whyte, A new method for the nonlinear transformation of means and covariances in filters and estimators. IEEE Trans. Autom. Control 45(3), 477-482 (2000)

8. M.H. Kabir, R. Kohno, A hybrid TOA-fingerprinting based localization of mobile nodes using UWB signaling for non line-of-sight conditions. Sensors 12(8), 11187-11204 (2012)

9. J. Li, B. Zhang, H. Liu, L. Yu, Z. Wang, An indoor hybrid localization approach based on signal propagation model and fingerprinting. Int. J. Smart Home 7(6), 157-170 (2013)

10. K.F. Li, Smart home technology for telemedicine and emergency management. J. Ambient Intell. Humanized Comput. 4(5), 535-546 (2013)

11. J. Prieto, S. Mazuelas, A. Bahillo, P. Fernández, R.M. Lorenzo, E.J. Abril, Adaptive data fusion for wireless localization in harsh environments. IEEE Tran. Sig. Proc. 60(4), 1585-1596 (2012) 
12. G. Villarrubia, J.F. de Paz, J. Bajo, J.M. Corchado, Monitoring and detection platform to prevent anomalous situations in home care. Sensors 14(6), 9900-9921 (2014)

13. F. Zampella, A. Bahillo, J. Prieto, A.R. Jiménez, F. Seco, Pedestrian navigation fusing inertial and RSS/TOF measurements with adaptive movement/measurement models: experimental evaluation and theoretical limits. Sens. Actuators A: Phys. 203, 249-260 (2013)

14. C. Zato, G. Villarrubia, A. Sánchez, I. Barri, E. Rubión, A. Fernández, C. Rebate, J.A. Cabo, T. Álamos, J. Sanz, J. Seco, J. Bajo, J.M. Corchado, PANGEA—platform for automatic coNstruction of orGanizations of intElligent agents, in Distributed Computing and Artificial Intelligence. Advances in Intelligent and Soft Computing, vol. 151 (Springer, Berlin, 2012), pp. 229-239 